Proceedings of the 16th International Conference

on Computational and Mathematical Methods

in Science and Engineering, CMMSE 2016

4-8 July, 2016.

\title{
Linear and Cyclic Codes over direct product of Finite Chain Rings
}

\author{
Joaquim Borges ${ }^{1}$, Cristina Fernández-Córdoba ${ }^{1}$ and Roger Ten-Valls ${ }^{1}$ \\ ${ }^{1}$ Department of Information and Communications Engineering, \\ Universitat Autònoma de Barcelona
}

emails: joaquim.borges@uab.cat, cristina.fernandez@uab.cat, roger.ten@uab.cat

\begin{abstract}
We introduce a new type of linear and cyclic codes. These codes are defined over a direct product of two finite chain rings. The definition of these codes as certain submodules of the direct product of copies of these rings is given and the cyclic property is defined. Cyclic codes can be seen as submodules of the direct product of polynomial rings. Generator matrices for linear codes and generator polynomials for cyclic codes are determined.
\end{abstract}

Key words: Codes over rings, linear codes, cyclic codes, finite chain rings

MSC 2000: 94B60, 94B25

\section{Introduction}

Linear codes are a special family of codes with rich mathematical structure. One of the most studied class of linear codes is the class of linear cyclic codes. The algebraic structure of cyclic codes makes easier their implementation. For this reason many practically important codes are cyclic.

The study of codes over rings has been growing since it was proven in [8] that certain notorious non-linear binary codes can be seen as binary images under the Gray map of linear codes over $\mathbb{Z}_{4}$. In particular, the family of codes over chain rings has received much attention because it includes some good codes (e.g. [6], [9]).

In recent times, linear codes with sets of coordinates over different rings are studied (e.g. $\mathbb{Z}_{2}^{\alpha} \times \mathbb{Z}_{4}^{\beta}$ in [3], $\mathbb{Z}_{p^{r}}^{\alpha} \times \mathbb{Z}_{p^{s}}^{\beta}$ in [2]). Also, linear cyclic codes over these kind of structures are studied, see [1], [4], [5] and [7]. 
In this paper we present the structure of linear and cyclic codes over direct product of finite chain rings, $\mathcal{R}_{1}$ and $\mathcal{R}_{2}$. Linear codes can be seen as certain submodules of $\mathcal{R}_{1}^{\alpha} \times \mathcal{R}_{2}^{\beta}$, and cyclic codes as submodules of $\mathcal{R}_{\alpha, \beta}=\frac{\mathcal{R}_{1}[x]}{\left\langle x^{\alpha}-1\right\rangle} \times \frac{\mathcal{R}_{2}[x]}{\left\langle x^{\beta}-1\right\rangle}$. We determine the generator matrix in standard form and the generator polynomials in the cyclic case. Finally, we present examples to illustrate some particular cases.

\section{Review of cyclic codes over finite chain rings}

Let $\mathcal{R}$ be a finite chain ring with maximal ideal $\langle\gamma\rangle$ and let $e$ be the nilpotency of $\gamma$. It is well-know that there exist a prime $p$ and a positive integer $m$ such that $|\mathcal{R} /\langle\gamma\rangle|=q=p^{m}$ and $|\mathcal{R}|=q^{e}=p^{m e}$.

Let $C$ be a cyclic code of length $n$ over $\mathcal{R}$. It is known that we can identify $C$ as an ideal of $\mathcal{R}[x] /\left(x^{n}-1\right)$. We assume that $n$ is a positive integer such that it is coprime with $p$. Therefore, the polynomial $x^{n}-1$ has a unique decomposition as a product of basic irreducible polynomials that are pairwise coprime over $\mathcal{R}[x]$.

Theorem 2.1 ([6, Theorem 3.5]). Let $C$ be a cyclic code of length $n$ over a finite chain ring $\mathcal{R}$, which has maximal ideal $\langle\gamma\rangle$ and $e$ is the nilpotency of $\gamma$. Then, there exist polynomials $g_{0}, g_{1}, \ldots, g_{e-1}$ in $\mathcal{R}[x]$ such that $C=\left\langle g_{0}, \gamma g_{1}, \ldots, \gamma^{e-1} g_{e-1}\right\rangle$ and $g_{e-1}\left|g_{e-2}\right| \cdots\left|g_{1}\right| g_{0} \mid$ $\left(x^{n}-1\right)$.

Let $C=\left\langle g_{0}, \gamma g_{1}, \ldots, \gamma^{e-1} g_{e-1}\right\rangle$ be a cyclic code of length $n$ and let $g=g_{0}+\gamma g_{1}+\cdots+$ $\gamma^{e-1} g_{e-1}$. Since $g_{0}$ is a factor of $x^{n}-1$ and for $i=1 \ldots e-1$ the polynomial $g_{i}$ is a factor of $g_{i-1}$, we will denote $\hat{g}_{0}=\frac{x^{n}-1}{g_{0}}$ and $\hat{g}_{i}=\frac{g_{i-1}}{g_{i}}$ for $i=1 \ldots e-1$. Define $G=\prod_{i=0}^{e-1} \hat{g}_{i}$, then it is clear that $G g=\left(\prod_{i=0}^{e-1} \hat{g}_{i}\right) g=0$ over $\mathcal{R}[x] /\left(x^{n}-1\right)$.

Lemma 2.2. Let $C$ be a cyclic code of length $n$ over a finite chain ring $\mathcal{R}$, which has maximal ideal $\langle\gamma\rangle$ and $e$ is the nilpotency of $\gamma$. Let $g_{0}, g_{1}, \ldots, g_{e-1}$ in $\mathcal{R}[x]$ such that $C=$ $\left\langle g_{0}, \gamma g_{1}, \ldots, \gamma^{e-1} g_{e-1}\right\rangle$ and $g_{e-1}\left|g_{e-2}\right| \cdots\left|g_{1}\right| g_{0} \mid\left(x^{n}-1\right)$. Then

$$
\begin{aligned}
& \text { 1. } \gamma^{e-1} g=\gamma^{e-1} g_{e-1} \frac{G}{\hat{g}_{0}}, \\
& \text { 2. } \gamma^{e-1-i}\left(\prod_{j=0}^{i-1} \hat{g}_{j}\right) g=\gamma^{e-1} g_{e-1} \frac{G}{\hat{g}_{i}}, \text { for } i=1, \ldots, e-1 \text {. }
\end{aligned}
$$

Proof. Let $g=g_{0}+\gamma g_{1}+\cdots+\gamma^{e-1} g_{e-1}$. Then

$$
\gamma^{e-1} g=\gamma^{e-1} g_{0} \frac{1}{g_{1}} \frac{g_{1}}{g_{2}} \ldots \frac{g_{e-3}}{g_{e-2}} \frac{g_{e-2}}{g_{e-1}} g_{e-1}=\gamma^{e-1} g_{e-1} \hat{g}_{1} \hat{g}_{2} \ldots \hat{g}_{e-2} \hat{g}_{e-1}=\gamma^{e-1} g_{e-1} \frac{G}{\hat{g}_{0}}
$$


and 1 holds. For $i=1, \ldots, e-1$ we have that

$$
\begin{aligned}
\gamma^{e-1-i}\left(\prod_{j=0}^{i-1} \hat{g}_{j}\right) g & =\gamma^{e-1-i}\left(\prod_{j=0}^{i-1} \hat{g}_{j}\right) \gamma^{i} g_{i} \frac{1}{g_{i+1}} \frac{g_{i+1}}{g_{i+2}} \ldots \frac{g_{e-2}}{g_{e-1}} g_{e-1} \\
& =\gamma^{e-1} g_{e-1} \hat{g}_{0} \hat{g}_{1} \ldots \hat{g}_{i-1} \hat{g}_{i+1} \ldots \hat{g}_{e-1}=\gamma^{e-1} g_{e-1} \frac{G}{\hat{g}_{i}}
\end{aligned}
$$

and statement 2 is proved.

Corollary 2.3 (of Th. 2.1). Let $C$ be a cyclic code of length $n$ over a finite chain ring $\mathcal{R}$ such that $C=\left\langle g_{0}, \gamma g_{1}, \ldots, \gamma^{e-1} g_{e-1}\right\rangle$ with $g_{e-1}\left|g_{e-2}\right| \cdots\left|g_{1}\right| g_{0} \mid\left(x^{n}-1\right)$. Then,

$$
|C|=|\mathcal{R} /\langle\gamma\rangle|^{\sum_{i=0}^{e-1}(e-i) \operatorname{deg}\left(\hat{g}_{i}\right)} .
$$

Proof. From the previous definition of $\hat{g}_{i}$, these polynomials are the same polynomials described in [6, Theorem 3.4].

Theorem 2.4. Let $C$ be a cyclic code of length $n$ over a finite chain ring $\mathcal{R}$, which has maximal ideal $\langle\gamma\rangle$ and $e$ is the nilpotency of $\gamma$. Let $g_{0}, g_{1}, \ldots, g_{e-1}$ polynomials in $\mathcal{R}[x]$ such that $C=\left\langle g_{0}, \gamma g_{1}, \ldots, \gamma^{e-1} g_{e-1}\right\rangle$ and $g_{e-1}\left|g_{e-2}\right| \cdots\left|g_{1}\right| g_{0} \mid\left(x^{n}-1\right)$. Then the polynomial $g=g_{0}+\gamma g_{1}+\cdots+\gamma^{e-1} g_{e-1}$ is a generating polynomial of $C$, i.e., $C=\langle g\rangle$.

Proof. Clearly $g=g_{0}+\gamma g_{1}+\cdots+\gamma^{e-1} g_{e-1} \in C$. then, we only have to prove that $\gamma^{i} g_{i} \in\langle g\rangle$, for all $i=0, \ldots, e-1$.

Case $e=2$ : We have that $g=g_{0}+\gamma g_{1}$, and $C=\left\langle g_{0}, \gamma g_{1}\right\rangle$. Then, $\gamma g=\gamma g_{0}=\gamma g_{1} \frac{g_{0}}{g_{1}}$ and $\frac{x^{n}-1}{g_{0}} g=\gamma g_{1} \frac{x^{n}-1}{g_{0}}$, since $\frac{g_{0}}{g_{1}}$ and $\frac{x^{n}-1}{g_{0}}$ are coprime then $\gamma g_{1}$ belongs to $\langle g\rangle$ and hence $g_{0}$ also belongs to $\langle g\rangle$.

Case $e=3$ : We have that $g=g_{0}+\gamma g_{1}+\gamma^{2} g_{2}$, and $C=\left\langle g_{0}, \gamma g_{1}, \gamma^{2} g_{2}\right\rangle$. Now $\gamma^{2} g=$ $\gamma^{2} g_{2} \frac{g_{1}}{g_{2}} \frac{g_{0}}{g_{1}}, \gamma \frac{x^{n}-1}{g_{0}} g=\gamma^{2} g_{2} \frac{x^{n}-1}{g_{0}} \frac{g_{1}}{g_{2}}$ and $\frac{x^{n}-1}{g_{0}} \frac{g_{0}}{g_{1}} g=\gamma^{2} g_{2} \frac{x^{n}-1}{g_{0}} \frac{g_{0}}{g_{1}}$, since $\operatorname{gcd}\left(\frac{g_{0}}{g_{1}}, \frac{g_{1}}{g_{2}}, \frac{x^{n}-1}{g_{0}}\right)=1$ then $\gamma^{2} g_{2}$ belongs to $\langle g\rangle$, hence $g_{0}+\gamma g_{1}$. So $\langle g\rangle=\left\langle g_{0}+\gamma g_{1}, \gamma^{2} g_{2}\right\rangle$. Arguing as in case $e=2$ it is straightforward that $\langle g\rangle=\left\langle g_{0}, \gamma g_{1}, \gamma^{2} g_{2}\right\rangle$.

In the general case, let $g=g_{0}+\gamma g_{1}+\cdots+\gamma^{e-1} g_{e-1}$ and define, as in Lemma 2.2, the polynomials $G$ and $\hat{g}_{i}$ for $i \in\{0, \ldots, e-1\}$. Then, $\gamma^{e-1} g=\gamma^{e-1} g_{e-1} \frac{G}{\hat{g}_{0}} \in\langle g\rangle$ and $\gamma^{e-1-i}\left(\prod_{j=0}^{i-1} \hat{g}_{j}\right) g=\gamma^{e-1} g_{e-1} \frac{G}{\hat{g}_{i}} \in\langle g\rangle$, for $i \in\{1, \ldots, e-1\}$. Since $\operatorname{gcd}\left(\frac{G}{\hat{g}_{0}}, \frac{G}{\hat{g}_{1}}, \ldots, \frac{G}{\hat{g}_{e-1}}\right)=1$, we have that $\gamma^{e-1} g_{e-1} \in\langle g\rangle$ and $\langle g\rangle=\left\langle g_{0}+\gamma g_{1}+\cdots+\gamma^{e-2} g_{e-2}, \gamma^{e-1} g_{e-1}\right\rangle$.

Reasoned similarly, one obtains that $\langle g\rangle=\left\langle g_{0}, \gamma g_{1}, \ldots, \gamma^{e-1} g_{e-1}\right\rangle$.

Theorem 2.5. Let $C=\langle g\rangle=\left\langle g_{0}+\gamma g_{1}+\cdots+\gamma^{e-2} g_{e-2}+\gamma^{e-1} g_{e-1}\right\rangle$ be a cyclic code of length $n$ over a finite chain ring $\mathcal{R}$, which has maximal ideal $\langle\gamma\rangle$ and $e$ is the nilpotency of 
$\gamma$ with $g_{e-1}\left|g_{e-2}\right| \cdots\left|g_{1}\right| g_{0} \mid\left(x^{n}-1\right)$. We define the sets

$$
S_{\gamma^{j}}=\left[x^{i}\left(\prod_{t=0}^{j-1} \hat{g}_{t}\right) g\right]_{i=0}^{\operatorname{deg}\left(\hat{g}_{j}\right)}
$$

for $0 \leq j<e$. Then,

$$
S=\bigcup_{j=0}^{e-1} S_{\gamma^{j}}
$$

forms a minimal generating set for $C$ as an $\mathcal{R}$-module.

Proof. Let $c \in \mathcal{C}$. Then, $c=d g$ with $d \in \mathcal{R}[x]$. If $\operatorname{deg}(d)<\operatorname{deg}\left(\hat{g}_{0}\right)$ then $d g \in\left\langle S_{\gamma^{0}}\right\rangle_{\mathcal{R}}$ and $c \in\langle S\rangle_{\mathcal{R}}$. Otherwise, compute $d=d_{0} \hat{g}_{0}+r_{0}$ with $\operatorname{deg}\left(r_{0}\right)<\operatorname{deg}\left(\hat{g}_{0}\right)$, so $d g=d_{0} \hat{g}_{0} g+r_{0} g$ and $r_{0} g \in\left\langle S_{\gamma^{0}}\right\rangle_{\mathcal{R}}$.

If $\operatorname{deg}\left(d_{0}\right)<\operatorname{deg}\left(\hat{g}_{1}\right)$ then $d_{0} \hat{g}_{0} g \in\left\langle S_{\gamma^{1}}\right\rangle_{\mathcal{R}}$ and $c \in\langle S\rangle_{\mathcal{R}}$. Otherwise, compute $d_{0}=$ $d_{1} \hat{g}_{1}+r_{1}$ with $\operatorname{deg}\left(r_{1}\right)<\operatorname{deg}\left(\hat{g}_{1}\right)$, so $d_{0} \hat{g}_{0} g=d_{1} \hat{g}_{1} \hat{g}_{0} g+r_{1} \hat{g}_{0} g$ and $r_{1} \hat{g}_{0} g \in\left\langle S_{\gamma^{1}}\right\rangle_{\mathcal{R}}$.

In the worst case and reasoning similarly, one obtains that $c \in\langle S\rangle_{\mathcal{R}}$ if $d_{e-2}\left(\prod_{t=0}^{e-2} \hat{g}_{t}\right) g \in$ $\langle S\rangle_{\mathcal{R}}$. It is obvious that if $\operatorname{deg}\left(d_{e-2}\right)<\operatorname{deg}\left(\hat{g}_{e-1}\right)$ then $d_{e-2}\left(\prod_{t=0}^{e-2} \hat{g}_{t}\right) g \in\left\langle S_{\gamma^{e-1}}\right\rangle_{\mathcal{R}}$, if not, $d_{e-2}=d_{e-1} \hat{g}_{e-1}+r_{e-1}$. Therefore,

$$
d_{e-2}\left(\prod_{t=0}^{e-2} \hat{g}_{t}\right) g=d_{e-1}\left(\prod_{t=0}^{e-1} \hat{g}_{t}\right) g+r_{e-1}\left(\prod_{t=0}^{e-2} \hat{g}_{t}\right) g=r_{e-1}\left(\prod_{t=0}^{e-2} \hat{g}_{t}\right) g \in\left\langle S_{\gamma^{e-1}}\right\rangle .
$$

Since $r_{e-1}\left(\prod_{t=0}^{e-2} \hat{g}_{t}\right) g \in\left\langle S_{\gamma^{e-1}}\right\rangle$ then $c \in\langle S\rangle_{\mathcal{R}}$, so $S$ is a generating set. By the definition of $S$ clearly

$$
|S|=|\mathcal{R} /\langle\gamma\rangle|^{\sum_{i=0}^{e-1}(e-i) \operatorname{deg}\left(\hat{g}_{i}\right)}
$$

By Corollary 2.3, $|C|=|S|$ and $S$ is a minimal generating set.

\section{Linear codes over $\mathcal{R}_{1}^{\alpha} \times \mathcal{R}_{2}^{\beta}$}

Let $\mathcal{R}_{1}$ and $\mathcal{R}_{2}$ be finite chain rings where $\gamma_{1}$ and $\gamma_{2}$ are generators of the maximal ideals of $\mathcal{R}_{1}$ and $\mathcal{R}_{2}$ with nilpotency indices $e_{1}$ and $e_{2}$, respectively. We will suppose that $\mathcal{R}_{1}$ and $\mathcal{R}_{2}$ have the same residue field $K=\mathcal{R}_{1} /\left\langle\gamma_{1}\right\rangle=\mathcal{R}_{2} /\left\langle\gamma_{2}\right\rangle$, with $|K|=q=p^{m}$. By ${ }^{-}: \mathcal{R}_{i} \rightarrow K$, we will denote the natural projection that maps $r \mapsto \bar{r}=r+\left\langle\gamma_{i}\right\rangle$, for $i=1$ or 2 .

Let $T_{1}=\left\{r_{0}, \ldots, r_{q-1}\right\}$ and $T_{2}=\left\{r_{0}^{\prime}, \ldots, r_{q-1}^{\prime}\right\}$ be the Teichmüller sets of representatives of $\mathcal{R}_{1}$ and $\mathcal{R}_{2}$, resp., then we can arrange the subscripts such that $\bar{r}_{i}=\bar{r}^{\prime}{ }_{i}$. Assume that $e_{1} \leq e_{2}$. Then we can consider the surjective ring homomorphism

$$
\begin{aligned}
\pi: \mathcal{R}_{2} & \rightarrow \mathcal{R}_{1} \\
\gamma_{2} & \mapsto \gamma_{1} \\
r_{j}^{\prime} & \mapsto r_{j}
\end{aligned}
$$




\section{J. Borges, C. Fernández-Córdoba, and R. Ten-Valls}

Note that $\pi\left(\gamma_{2}{ }^{i}\right)=0$ if $i \geq e_{1}$. For $a \in \mathcal{R}_{2}$ and $b \in \mathcal{R}_{1}$, define a multiplication * as follows: $a * b=\pi(a) b$. Then, $\mathcal{R}_{1}$ is an $\mathcal{R}_{2}$-module with external multiplication $*$ given by $\pi$. Since $\mathcal{R}_{1}$ is commutative then $*$ has the commutative property. Then, we can generalize this multiplication over the ring $\mathcal{R}_{1}^{\alpha} \times \mathcal{R}_{2}^{\beta}$ as follows. Let $a$ be an element of $\mathcal{R}_{2}$ and $\mathbf{u}=\left(u \mid u^{\prime}\right)=\left(u_{0}, u_{1}, \ldots, u_{\alpha-1} \mid u_{0}^{\prime}, u_{1}^{\prime}, \ldots, u_{\beta-1}^{\prime}\right) \in \mathcal{R}_{1}^{\alpha} \times \mathcal{R}_{2}^{\beta}$. Then,

$$
a * \mathbf{u}=\left(\pi(a) u_{0}, \pi(a) u_{1}, \ldots, \pi(a) u_{\alpha-1} \mid a u_{0}^{\prime}, a u_{1}^{\prime}, \ldots, a u_{\beta-1}^{\prime}\right) .
$$

With this external operation the ring $\mathcal{R}_{1}^{\alpha} \times \mathcal{R}_{2}^{\beta}$ is also an $\mathcal{R}_{2}$-module.

Definition 3.1. A subset $\mathcal{C} \subseteq \mathcal{R}_{1}^{\alpha} \times \mathcal{R}_{2}^{\beta}$ is a linear code if it is a submodule of $\mathcal{R}_{1}^{\alpha} \times \mathcal{R}_{2}^{\beta}$.

The next result gives the structure of a generator matrix of a linear code.

Proposition 3.2. Let $\mathcal{C}$ be a linear code over $\mathcal{R}_{1}^{\alpha} \times \mathcal{R}_{2}^{\beta}$. Then $\mathcal{C}$ is permutation equivalent to a code with generator matrix of the form

$$
G=\left(\begin{array}{c|c}
B & T \\
S & A
\end{array}\right)
$$

where

$$
\begin{aligned}
& B=\left(\begin{array}{ccccccc}
I_{k_{0}} & B_{0,1} & B_{0,2} & B_{0,3} & \ldots & B_{0, e_{1}-1} & B_{0, e_{1}} \\
0 & \gamma_{1} I_{k_{1}} & \gamma_{1} B_{1,2} & \gamma_{1} B_{1,3} & \ldots & \gamma_{1} B_{1, e_{1}-1} & \gamma_{1} B_{1, e_{1}} \\
0 & 0 & \gamma_{1}^{2} I_{k_{2}} & \gamma_{1}^{2} B_{2,3} & \ldots & \gamma_{1}^{2} B_{2, e_{1}-1} & \gamma_{1}^{2} B_{2, e_{1}} \\
\vdots & \vdots & \vdots & \vdots & & \vdots & \vdots \\
0 & 0 & 0 & 0 & \ldots & \gamma_{1}^{e_{1}-1} I_{k_{e_{1}-1}} & \gamma_{1}^{e_{1}-1} B_{e_{1}-1, e_{1}}
\end{array}\right), \\
& T=\left(\begin{array}{ccccc}
0 \ldots & \gamma_{2}^{e_{2}-e_{1}} T_{0,1} & \gamma_{2}^{e_{2}-e_{1}} T_{0,2} & \ldots & \gamma_{2}^{e_{2}-e_{1}} T_{0, e_{1}} \\
0 \ldots & 0 & \gamma_{2}^{e_{2}-e_{1}+1} T_{1,2} & \ldots & \gamma_{2}^{e_{2}-e_{1}+1} T_{1, e_{1}} \\
\vdots & \vdots & \vdots & & \vdots \\
0 & 0 & 0 & \ldots & \gamma_{2}^{e_{2}-1} T_{e_{1}-1, e_{1}}
\end{array}\right) \\
& A=\left(\begin{array}{ccccccc}
I_{l_{0}} & A_{0,1} & A_{0,2} & A_{0,3} & \ldots & A_{0, e_{1}-1} & A_{0, e_{2}} \\
0 & \gamma_{2} I_{l_{1}} & \gamma_{2} A_{1,2} & \gamma_{2} A_{1,3} & \ldots & \gamma_{2} A_{1, e_{2}-1} & \gamma_{2} A_{1, e_{2}} \\
0 & 0 & \gamma_{2}^{2} I_{l_{2}} & \gamma_{2}^{2} A_{2,3} & \ldots & \gamma_{2}^{2} A_{2, e_{2}-1} & \gamma_{2}^{2} A_{2, e_{2}} \\
\vdots & \vdots & \vdots & \vdots & & \vdots & \vdots \\
0 & 0 & 0 & 0 & \ldots & \gamma_{2}^{e_{2}-1} I_{l_{e_{2}-1}} & \gamma_{2}^{e_{2}-1} A_{e_{2}-1, e_{2}}
\end{array}\right) \text {, } \\
& S=\left(\begin{array}{cccccc}
0 & S_{0,1} & S_{0,2} & \ldots & S_{0, e_{1}-1} & S_{0, e_{1}} \\
0 & S_{1,1} & S_{1,2} & \ldots & S_{1, e_{1}-1} & S_{1, e_{1}} \\
\vdots & \vdots & \vdots & & \vdots & \vdots \\
0 & S_{e_{2}-e_{1}-1,1} & S_{e_{2}-e_{1}-1,2} & \ldots & S_{e_{2}-e_{1}-1, e_{1}-1} & S_{e_{2}-e_{1}-1, e_{1}} \\
0 & 0 & \gamma_{1} S_{e_{2}-e_{1}, 2} & \ldots & \gamma_{1} S_{e_{2}-e_{1}, e_{1}-1} & \gamma_{1} S_{e_{2}-e_{1}, e_{1}} \\
\vdots & \vdots & \vdots & & \vdots & \vdots \\
0 & 0 & 0 & \ldots & \gamma_{1}^{e_{1}-2} S_{e_{2}-3, e_{1}-1} & \gamma_{1}^{e_{1}-3} S_{e_{2}-2, e_{1}} \\
0 & 0 & 0 & \ldots & 0 & \gamma_{1}^{e_{1}-1} S_{e_{2}-2, e_{1}} \\
0 & 0 & 0 & \ldots & 0 & 0
\end{array}\right)
\end{aligned}
$$

where the entries in $\gamma_{1}^{i} B_{i, j}$ and $\gamma_{1}^{i} S_{i, j}$ are in $\left\langle\gamma_{1}^{i}\right\rangle$ and the ones in $\gamma_{2}^{t} A_{t, j}$ and $\gamma_{2}^{t} T_{t, j}$ are in $\left\langle\gamma_{2}^{t}\right\rangle$. 
Proof. Similiar to [2, Theorem 4]

Let $\mathcal{C}_{X}$ be the canonical projection of $\mathcal{C}$ on the first $\alpha$ coordinates and $\mathcal{C}_{Y}$ on the last $\beta$ coordinates. The canonical projection is a linear map. Then, $\mathcal{C}_{X}$ and $\mathcal{C}_{Y}$ are $\mathcal{R}_{1}$ and $\mathcal{R}_{2}$ linear codes of length $\alpha$ and $\beta$, respectively. A code $\mathcal{C}$ is called separable if $\mathcal{C}$ is the direct product of $\mathcal{C}_{X}$ and $\mathcal{C}_{Y}$, i.e., $\mathcal{C}=\mathcal{C}_{X} \times \mathcal{C}_{Y}$. Moreover, if $\mathcal{C}$ is separable then

$$
G=\left(\begin{array}{c|c}
B & 0 \\
0 & A
\end{array}\right)
$$

Example 1. Let $\mathcal{C}$ be a linear code over $\mathbb{Z}_{2}^{3} \times\left(\frac{\mathbb{Z}_{2}[u]}{\left\langle u^{3}\right\rangle}\right)^{4}$ generated by the matrix

$$
\left(\begin{array}{ccc|cccc}
1 & 1 & 0 & u & u+u^{2} & 1+u & 1+u^{2} \\
0 & 1 & 0 & 1 & u & u^{2} & 0 \\
0 & 1 & 1 & 0 & u^{2} & 0 & u^{2} \\
1 & 1 & 1 & u^{2} & u & u+u^{2} & 0
\end{array}\right)
$$

Hence, as described in Theorem 3.2, $\mathcal{C}$ is permutation equivalent to a code generated by the following matrix:

$$
G=\left(\begin{array}{ccc|cccc}
1 & 1 & 1 & 0 & 0 & 0 & 0 \\
\hline 0 & 0 & 1 & 1 & 0 & 0 & u \\
0 & 1 & 0 & 0 & 1 & 0 & 1 \\
0 & 1 & 1 & 0 & 0 & u & u+u^{2}
\end{array}\right)
$$

Note that $\mathcal{C}$ is not separable.

\section{Cyclic codes over $\mathcal{R}_{1}^{\alpha} \times \mathcal{R}_{2}^{\beta}$}

Definition 4.1. Let $\mathcal{C}$ be a linear code over $\mathcal{R}_{1}^{\alpha} \times \mathcal{R}_{2}^{\beta}$. The code $\mathcal{C}$ is called cyclic if

$$
\left(u_{0}, u_{1}, \ldots, u_{\alpha-2}, u_{\alpha-1} \mid u_{0}^{\prime}, u_{1}^{\prime}, \ldots, u_{\beta-2}^{\prime}, u_{\beta-1}^{\prime}\right) \in \mathcal{C}
$$

implies

$$
\left(u_{\alpha-1}, u_{0}, u_{1}, \ldots, u_{\alpha-2} \mid u_{\beta-1}^{\prime}, u_{0}^{\prime}, u_{1}^{\prime}, \ldots, u_{\beta-2}^{\prime}\right) \in \mathcal{C} .
$$

Let $\mathbf{u}=\left(u_{0}, u_{1}, \ldots, u_{\alpha-1} \mid u_{0}^{\prime}, \ldots, u_{\beta-1}^{\prime}\right)$ be a codeword in $\mathcal{C}$ and let $i$ be an integer. We then denote by $\mathbf{u}^{(i)}=\left(u_{0+i}, u_{1+i}, \ldots, u_{\alpha-1+i} \mid u_{0+i}^{\prime}, \ldots, u_{\beta-1+i}^{\prime}\right)$ the $i$ th shift of $\mathbf{u}$, where the subscripts are read modulo $\alpha$ and $\beta$, respectively.

We remark that in this paper the definition of a cyclic code over $\mathcal{R}_{1}^{\alpha} \times \mathcal{R}_{2}^{\beta}$ is clear as long as $\mathcal{R}_{1}$ and $\mathcal{R}_{2}$ are different rings, since the elements on the first $\alpha$ coordinates and the ones in the last $\beta$ coordinates belong from different rings, $\mathcal{R}_{1}$ and $\mathcal{R}_{2}$, respectively. In the 


\section{J. Borges, C. Fernández-Córdoba, and R. Ten-Valls}

particular case that $\mathcal{R}_{1}=\mathcal{R}_{2}$, the cyclic code over $\mathcal{R}_{1}^{\alpha} \times \mathcal{R}_{2}^{\beta}$ is known in the literature as double cyclic code, see [4], [7]. The term double cyclic is given in order to distinguish the cyclic code over $\mathcal{R}_{1}^{\alpha} \times \mathcal{R}_{1}^{\beta}$ from the cyclic code over $\mathcal{R}_{1}^{\alpha+\beta}$.

Note that $\mathcal{C}_{X}$ and $\mathcal{C}_{Y}$ are $\mathcal{R}_{1}$ and $\mathcal{R}_{2}$ cyclic codes of length $\alpha$ and $\beta$, respectively.

Denote by $\mathcal{R}_{\alpha, \beta}$ the ring $\mathcal{R}_{1}[x] /\left(x^{\alpha}-1\right) \times \mathcal{R}_{2}[x] /\left(x^{\beta}-1\right)$. There is a bijective map between $\mathcal{R}_{1}^{\alpha} \times \mathcal{R}_{2}^{\beta}$ and $\mathcal{R}_{\alpha, \beta}$ where $\mathbf{u}=\left(u_{0}, u_{1}, \ldots, u_{\alpha-1} \mid u_{0}^{\prime}, \ldots, u_{\beta-1}^{\prime}\right)$ maps to $\mathbf{u}(x)=$ $\left(u_{0}+u_{1} x+\cdots+u_{\alpha-1} x^{\alpha-1} \mid u_{0}^{\prime}+\cdots+u_{\beta-1}^{\prime} x^{\beta-1}\right)$.

Note that we can extend the map $\pi$ to the polynomial ring $\mathcal{R}_{2}[x]$ applying this map to each of the coefficients of a given polynomial.

Definition 4.2. Define the operation $*: \mathcal{R}_{2}[x] \times \mathcal{R}_{\alpha, \beta} \rightarrow \mathcal{R}_{\alpha, \beta}$ as

$$
\lambda(x) *(p(x) \mid q(x))=(\pi(\lambda(x)) p(x) \mid \lambda(x) q(x)),
$$

where $\lambda(x) \in \mathcal{R}_{2}[x]$ and $(p(x) \mid q(x)) \in \mathcal{R}_{\alpha, \beta}$.

The ring $\mathcal{R}_{\alpha, \beta}$ with the external operation $*$ is a $\mathcal{R}_{2}[x]$-module. Let $\mathbf{u}(x)=\left(u(x) \mid u^{\prime}(x)\right)$ be an element of $\mathcal{R}_{\alpha, \beta}$. Note that if we operate $\mathbf{u}(x)$ by $x$ we get

$$
\begin{aligned}
x * \mathbf{u}(x)=x *\left(u(x) \mid u^{\prime}(x)\right) & =\left(u_{0} x+\cdots+u_{\alpha-1} x^{\alpha} \mid u_{0}^{\prime} x+\cdots+u_{\beta-1}^{\prime} x^{\beta}\right) \\
& =\left(u_{\alpha-1}+\cdots+u_{\alpha-2} x^{\alpha-1} \mid u_{\beta-1}^{\prime}+\cdots+u_{\beta-2}^{\prime} x^{\beta-1}\right) .
\end{aligned}
$$

Hence, $x * \mathbf{u}(x)$ is the image of the vector $\mathbf{u}^{(1)}$. Thus, the operation of $\mathbf{u}(x)$ by $x$ in $R_{\alpha, \beta}$ corresponds to a shift of $\mathbf{u}$. In general, $x^{i} * \mathbf{u}(x)=\mathbf{u}^{(i)}(x)$ for all $i$.

\subsection{Algebraic structure and generators of cyclic codes}

In this subsection, we study submodules of $\mathcal{R}_{\alpha, \beta}$. We describe the generators of such submodules and state some properties. From now on, $\langle S\rangle$ will denote the $\mathcal{R}_{2}[x]$-submodule generated by a subset $S$ of $\mathcal{R}_{\alpha, \beta}$.

For the rest of the discussion we will consider that $\alpha$ and $\beta$ are coprime integers with $p$. From this assumption, we know that $\mathcal{R}_{1}[x] /\left\langle x^{\alpha}-1\right\rangle$ and $\mathcal{R}_{2}[x] /\left\langle x^{\beta}-1\right\rangle$ are principal ideal rings, see [6].

Theorem 4.3. Every submodule $\mathcal{C}$ of the $\mathcal{R}_{2}[x]$-module $\mathcal{R}_{\alpha, \beta}$ can be written as

$$
\mathcal{C}=\langle(b(x) \mid 0),(\ell(x) \mid a(x))\rangle
$$

where $b(x), a(x)$ are generator polynomials in $\mathcal{R}_{1}[x] /\left(x^{\alpha}-1\right)$ and $\mathcal{R}_{2}[x] /\left(x^{\beta}-1\right)$ resp., and $\ell(x) \in \mathcal{R}_{1}[x] /\left(x^{\alpha}-1\right)$. 
Proof. Let $\psi_{X}: \mathcal{R}_{\alpha, \beta} \rightarrow \mathcal{R}_{1}[x] /\left(x^{\alpha}-1\right)$ and $\psi_{Y}: \mathcal{R}_{\alpha, \beta} \rightarrow \mathcal{R}_{2}[x] /\left(x^{\beta}-1\right)$ be the canonical projections, let $\mathcal{C}$ be a submodule of $\mathcal{R}_{\alpha, \beta}$.

Define $\mathcal{C}^{\prime}=\{(p(x) \mid q(x)) \in \mathcal{C} \mid q(x)=0\}$. It is easy to check that $\mathcal{C}^{\prime} \cong \psi_{X}\left(\mathcal{C}^{\prime}\right)$ by $(p(x) \mid$ $0) \mapsto p(x)$. Hence, by Theorem $2.4, \psi_{X}\left(\mathcal{C}^{\prime}\right)$ is finitely generated by one element and so is $\mathcal{C}^{\prime}$. Let $b(x)$ be a generator of $\psi_{X}\left(\mathcal{C}^{\prime}\right)$, then $(b(x) \mid 0)$ is a generator of $\mathcal{C}^{\prime}$.

As $\mathcal{R}_{2}[x] /\left(x^{\beta}-1\right)$ is also a principal ideal ring, then $\mathcal{C}_{Y}=\psi_{Y}(\mathcal{C})$ is generated by one element. Let $a(x) \in \mathcal{C}_{Y}$ such that $\mathcal{C}_{Y}=\langle a(x)\rangle$, then there exists $\ell(x) \in \mathcal{R}_{1}[x] /\left(x^{\alpha}-1\right)$ such that $(\ell(x) \mid a(x)) \in \mathcal{C}$.

We claim that

$$
\mathcal{C}=\langle(b(x) \mid 0),(\ell(x) \mid a(x))\rangle .
$$

Let $(p(x) \mid q(x)) \in \mathcal{C}$, then $q(x)=\psi_{Y}(p(x) \mid q(x)) \in \mathcal{C}_{Y}$. So, there exists $\lambda(x) \in \mathcal{R}_{2}[x]$ such that $q(x)=\lambda(x) a(x)$. Now,

$$
(p(x) \mid q(x))-\lambda(x) *(\ell(x) \mid a(x))=(p(x)-\pi(\lambda(x)) \ell(x) \mid 0)
$$

belongs to $\mathcal{C}^{\prime}$. Then, there exists $\mu(x) \in \mathcal{R}_{2}[x]$ such that $(p(x)-\pi(\lambda(x)) \ell(x) \mid 0)=$ $\mu(x) *(b(x) \mid 0)$. Thus,

$$
(p(x) \mid q(x))=\mu(x) *(b(x) \mid 0)+\lambda(x) *(\ell(x) \mid a(x)) .
$$

So, $\mathcal{C}$ is finitely generated by $\langle(b(x) \mid 0),(\ell(x) \mid a(x))\rangle$.

From the previous results, it is clear that we can identify double cyclic codes in $\mathcal{R}_{1}^{\alpha} \times \mathcal{R}_{2}^{\beta}$ as submodules of $\mathcal{R}_{\alpha, \beta}$. So, any submodule of $\mathcal{R}_{\alpha, \beta}$ is a cyclic code. From now on, we will denote by $\mathcal{C}$ indistinctly both the code and the corresponding submodule of $\mathcal{R}_{\alpha, \beta}$.

In the following, a polynomial $f(x)$ in $\mathcal{R}_{1}[x]$ or $\mathcal{R}_{2}[x]$ will be denoted simply by $f$.

Proposition 4.4. Let $\mathcal{C}$ be a cyclic code over $\mathcal{R}_{1}^{\alpha} \times \mathcal{R}_{2}^{\beta}$. Then, there exist polynomials $\ell$ and $b_{e_{1}-1}\left|b_{e_{1}-2}\right| \ldots\left|b_{1}\right| b_{0} \mid\left(x^{\alpha}-1\right)$ over $\mathcal{R}_{1}[x]$ and $a_{e_{2}-1}\left|a_{e_{2}-2}\right| \ldots\left|a_{1}\right| a_{0} \mid\left(x^{\beta}-1\right)$ over $\mathcal{R}_{2}[x]$ such that

$$
\mathcal{C}=\left\langle\left(b_{0}+\gamma_{1} b_{1}+\cdots+\gamma_{1}^{e_{1}-1} b_{e_{1}-1} \mid 0\right),\left(\ell \mid a_{0}+\gamma_{2} a_{1}+\cdots+\gamma_{2}{ }^{e_{2}-1} a_{e_{2}-1}\right)\right\rangle
$$

Proof. Let $\mathcal{C}$ be a cyclic code over $\mathcal{R}_{1}^{\alpha} \times \mathcal{R}_{2}^{\beta}$. By Theorem 4.3, there exist polynomials $b, \ell \in \mathcal{R}_{1}[x] /\left(x^{\alpha}-1\right)$ and $a \in \mathcal{R}_{2}[x] /\left(x^{\beta}-1\right)$ such that $\mathcal{C}=\langle(b \mid 0),(\ell \mid a)\rangle$. By Theorem 2.4 , one can consider $b=b_{0}+\gamma_{1} b_{1}+\cdots+\gamma_{1}{ }^{e_{1}-1} b_{e_{1}-1}$ and $a=a_{0}+\gamma_{2} a_{1}+\cdots+\gamma_{2}{ }^{e_{2}-1} a_{e_{2}-1}$ such that $b_{e_{1}-1}\left|b_{e_{1}-2}\right| \ldots\left|b_{1}\right| b_{0} \mid\left(x^{\alpha}-1\right)$ and $a_{e_{2}-1}\left|a_{e_{2}-2}\right| \ldots\left|a_{1}\right| a_{0} \mid\left(x^{\beta}-1\right)$.

For the rest of the discussion, any cyclic code $\mathcal{C}$ over $\mathcal{R}_{1}^{\alpha} \times \mathcal{R}_{2}^{\beta}$ is of the form $\mathcal{C}=\langle(b \mid$ $0),(\ell \mid a)\rangle$, where $b=b_{0}+\gamma_{1} b_{1}+\cdots+\gamma_{1}{ }^{e_{1}-1} b_{e_{1}-1}$ and $a(x)=a_{0}+\gamma_{2} a_{1}+\cdots+\gamma_{2}{ }^{e_{2}-1} a_{e_{2}-1}$, for polynomials $b_{i}$ and $a_{j}$ as in Proposition 4.4. 


\section{J. Borges, C. Fernández-Córdoba, and R. Ten-Valls}

Example 2. Let $\mathcal{R}_{1}=\frac{\mathbb{F}_{9}[u]}{\left\langle u^{2}\right\rangle}$ and $\mathcal{R}_{2}=\frac{\mathbb{F}_{9}[u]}{\left\langle u^{3}\right\rangle}$, with $\gamma_{1}=u, e_{1}=2, \gamma_{2}=u$, and $e_{2}=3$. Let $\xi$ be a generator of the multiplicative group $\mathbb{F}_{9}^{*}$. Consider the cyclic code over $\left(\frac{\mathbb{F}_{9}[u]}{\left\langle u^{2}\right\rangle}\right)^{4} \times$ $\left(\frac{\mathbb{F}_{9}[u]}{\left\langle u^{3}\right\rangle}\right)^{10}$ generated by

$$
\mathcal{C}=\left\langle\left(u\left(x^{2}-1\right) \mid 0\right),\left(u \mid\left(x^{4}+\xi^{3} x^{2}+1\right)+u\left(x^{2}+\xi^{5} x+1\right)+u^{2}\right)\right\rangle .
$$

Then,

$$
b_{0}=x^{4}-1, \quad b_{1}=x^{2}-1, \quad \ell=u, a_{0}=x^{4}+\xi^{3} x^{2}+1, \quad a_{1}=x^{2}+\xi^{5} x+1, \quad a_{2}=1 .
$$

\subsection{Minimal generating sets}

Our goal is to find a set generators for a cyclic code, $\mathcal{C}$, as an $\mathcal{R}_{2}$-module. Once we found it, we are going to use it to determine the size of $\mathcal{C}$ in terms of the generator polynomials.

Since $b_{0}$ is a factor of $x^{\alpha}-1$ and for $i=1 \ldots e_{1}-1$ the polynomial $b_{i}$ is a factor of $b_{i-1}$, we will denote $\hat{b}_{0}=\frac{x^{\alpha}-1}{b_{0}}, \hat{b}_{i}=\frac{b_{i-1}}{b_{i}}$ for $i=1 \ldots e_{1}-1$, and $\hat{b}_{e_{1}}=b_{e_{1}-1}$. In the same way, we define $\hat{a}_{0}=\frac{x^{\beta}-1}{a_{0}}, \hat{a}_{j}=\frac{a_{j-1}}{a_{j}}$ for $j=1 \ldots e_{2}-1$, and $\hat{a}_{e_{2}}=a_{e_{2}-1}$.

Theorem 4.5. Let $\mathcal{C}$ be a cyclic code over $\mathcal{R}_{1}^{\alpha} \times \mathcal{R}_{2}^{\beta}$ which has maximals ideals $\left\langle\gamma_{1}\right\rangle \subset \mathcal{R}_{1}$ and $\left\langle\gamma_{2}\right\rangle \subset \mathcal{R}_{2}$ with nilpotent indices $e_{1}$ and $e_{2}$, respectively. Define

$$
B_{j}=\left[x^{i}\left(\prod_{t=0}^{j-1} \hat{b}_{t}\right) *(b \mid 0)\right]_{i=0}^{\operatorname{deg}\left(\hat{b}_{j}\right)-1},
$$

for $0 \leq j<e_{1}$, and

$$
A_{k}=\left[x^{i}\left(\prod_{t=0}^{k-1} \hat{a}_{t}\right) *(\ell \mid a)\right]_{i=0}^{\operatorname{deg}\left(\hat{a}_{k}\right)-1}
$$

for $0 \leq k<e_{2}$. Then,

$$
S=\left(\bigcup_{j=0}^{e_{1}-1} B_{j}\right) \bigcup\left(\bigcup_{k=0}^{e_{2}-1} A_{k}\right)
$$

forms a minimal generating set for $\mathcal{C}$ as an $\mathcal{R}_{2}$-module. Moreover,

$$
|\mathcal{C}|=q^{\sum_{i=0}^{e_{1}-1}\left(e_{1}-i\right) \operatorname{deg}\left(\hat{b}_{i}\right)+\sum_{j=0}^{e_{2}-1}\left(e_{2}-j\right) \operatorname{deg} \hat{a}_{j}}
$$

where $q$ is the cardinality of the residue field. 
Proof. By Theorem 2.5, it is clear that the elements in $S$ are $\mathcal{R}_{2}$-lineal independent since $\left(\bigcup_{j=0}^{e_{1}-1} B_{j}\right)_{X}$ and $\left(\bigcup_{k=0}^{e_{2}-1} A_{k}\right)_{Y}$ are minimal generating sets for the codes $\mathcal{C}_{X}$ and $\mathcal{C}_{Y}$, respectively. Let $c$ be a codeword of $\mathcal{C}$, then $c=q *(b \mid 0)+d *(\ell \mid a)$. Reasoning similarly as in Theorem 2.5, we have that $q *(b \mid 0) \in\left\langle\bigcup_{j=0}^{e_{1}-1} B_{j}\right\rangle_{\mathcal{R}_{2}}$. So, we have to prove that $d *(\ell \mid a) \in\langle S\rangle_{\mathcal{R}_{2}}$.

If $\operatorname{deg}(d)<\operatorname{deg}\left(\hat{a}_{0}\right)$ then $d *(\ell \mid a) \in\left\langle A_{0}\right\rangle_{\mathcal{R}_{2}}$ and $c \in\langle S\rangle_{\mathcal{R}_{2}}$. Otherwise, compute $d=d_{0} \hat{a}_{0}+r_{0}$ with $\operatorname{deg}\left(r_{0}\right)<\operatorname{deg}\left(\hat{a}_{0}\right)$, so $d *(\ell \mid a)=d_{0} \hat{a}_{0} *(\ell \mid a)+r_{0} *(\ell \mid a)$ and $r_{0} *(\ell \mid a) \in\left\langle A_{0}\right\rangle_{\mathcal{R}_{2}}$.

In the worst case and reasoning similarly, one obtains that $c \in\langle S\rangle_{\mathcal{R}_{2}}$ if $d_{e_{2}-2}\left(\prod_{t=0}^{e_{2}-2} \hat{a}_{t}\right) *$ $(\ell \mid a) \in\langle S\rangle_{\mathcal{R}_{2}}$. It is obvious that if $\operatorname{deg}\left(d_{e_{2}-2}\right)<\operatorname{deg}\left(\hat{a}_{e_{2}-1}\right)$ then $d_{e_{2}-2}\left(\prod_{t=0}^{e_{2}-2} \hat{a}_{t}\right) *(\ell \mid$ a) $\in\left\langle A_{e_{2}-1}\right\rangle_{\mathcal{R}_{2}}$, if not $d_{e_{2}-2}=d_{e_{2}-1} \hat{a}_{e_{2}-1}+r_{e_{2}-1}$. Therefore,

$$
d_{e_{2}-2}\left(\prod_{t=0}^{e_{2}-2} \hat{a}_{t}\right) *(\ell \mid a)=d_{e_{2}-1}\left(\prod_{t=0}^{e_{2}-1} \hat{a}_{t}\right) *(\ell \mid a)+r_{e_{2}-1}\left(\prod_{t=0}^{e_{2}-2} \hat{a}_{t}\right) *(\ell \mid a) .
$$

On one hand, $r_{e_{2}-1}\left(\prod_{t=0}^{e_{2}-2} \hat{a}_{t}\right) *(\ell \mid a) \in\left\langle A_{e_{2}-1}\right\rangle_{\mathcal{R}_{2}}$. On the other hand, $d_{e_{2}-1}\left(\prod_{t=0}^{e_{2}-1} \hat{a}_{t}\right) *(\ell \mid$ $a)=d_{e_{2}-1}\left(\prod_{t=0}^{e_{2}-1} \hat{a}_{t}\right) *(\ell \mid 0)$ and then $d_{e_{2}-1}\left(\prod_{t=0}^{e_{2}-1} \hat{a}_{t}\right) *(\ell \mid a)=f *(b \mid 0) \in\left\langle\bigcup_{j=0}^{e_{1}-1} B_{j}\right\rangle_{\mathcal{R}_{2}}$. Thus, $c \in\langle S\rangle_{\mathcal{R}_{2}}$ and $S$ is a minimal generating set for $\mathcal{C}$.

Example 3. Consider $\mathcal{R}_{\alpha, \beta}=\mathbb{Z}_{2}[x] /\left(x^{2}-1\right) \times \mathbb{Z}_{4}[x] /\left(x^{3}-1\right)$ and the cyclic code

$$
\mathcal{C}=\left\langle\left(x-1 \mid\left(x^{2}+x+1\right)+2\right)\right\rangle,
$$

where $b_{0}(x)=x^{2}-1, \ell(x)=x-1, a_{0}(x)=x^{2}+x+1$ and $a_{1}(x)=1$. Then, $S=\{(x-1 \mid$ $\left.\left.x^{2}+x+3\right),(0 \mid 2 x+2),\left(0 \mid 2 x^{2}+2 x\right)\right\}$ and

$$
|\mathcal{C}|=2^{\sum_{j=0}^{2-1}(2-j) \operatorname{deg}\left(\hat{a}_{j}\right)}=2^{4}=16 .
$$

Example 4. From Example 2, consider the cyclic code over $\left(\frac{\mathbb{F}_{9}[u]}{\left\langle u^{2}\right\rangle}\right)^{4} \times\left(\frac{\mathbb{F}_{9}[u]}{\left\langle u^{3}\right\rangle}\right)^{10}$ generated by

$$
\mathcal{C}=\left\langle\left(u\left(x^{2}-1\right) \mid 0\right),\left(u \mid\left(x^{4}+\xi^{3} x^{2}+1\right)+u\left(x^{2}+\xi^{7} x+1\right)+u^{2}\right)\right\rangle .
$$

Let $b=u\left(x^{2}-1\right), \ell=u$ and $a=\left(x^{4}+\xi^{3} x^{2}+1\right)+u\left(x^{2}+\xi^{7} x+1\right)+u^{2}$. Then, a minimal generating set for $\mathcal{C}$ is the union of

$$
\begin{gathered}
B_{0}=\emptyset, B_{1}=\left[x^{i} *\left(u\left(x^{2}-1\right) \mid 0\right)\right]_{i=0}^{1}, \\
A_{0}=\left[x^{i} *(\ell \mid a)\right]_{i=0}^{5}, A_{1}=\left[x^{i} \mu *(\ell \mid a)\right]_{i=0}^{1},
\end{gathered}
$$

and

$$
A_{2}=\left[x^{i} \lambda *(\ell \mid a)\right]_{i=0}^{1},
$$

where $\mu=x^{6}+\xi^{7} x^{4}+\xi^{3} x^{2}+2$ and $\lambda=x^{8}+\xi x^{7}+\xi x^{6}+x^{5}+2 x^{3}+\xi^{5} x^{2}+\xi^{5} x+2$. So,

$$
|\mathcal{C}|=9^{\sum_{i=0}^{2-1}(2-i) \operatorname{deg}\left(\hat{b}_{i}\right)+\sum_{j=0}^{3-1}(3-j) \operatorname{deg}\left(\hat{a}_{j}\right)}=9^{2+24}=9^{26} .
$$


J. Borges, C. Fernández-Córdoba, and R. Ten-Valls

\section{Acknowledgements}

This work has been partially supported by the Spanish MINECO grants TIN2013-40524-P and MTM2015-69138-REDT, and by the Catalan AGAUR grant 2014SGR-691.

\section{References}

[1] T. Abualrub, I. Siap, N. Aydin, $\mathbb{Z}_{2} \mathbb{Z}_{4}$-additive cyclic codes, IEEE Trans. Info. Theory 60(3) (2014) 1508-1514.

[2] I. Aydogdu, I. Siap, On $\mathbb{Z}_{p^{r}} \mathbb{Z}_{p^{s}}$-additive codes, Linear and Multilinear Algebra 63(10) (2014) 2089-2102.

[3] J. Borges, C. Fernández-Córdoba, J. Pujol, J. Rifà and M. Villanueva, $\mathbb{Z}_{2} \mathbb{Z}_{4}$-linear codes: generator matrices and duality, Des., Codes and Crypto. 54(2) (2010) 167-179.

[4] J. Borges, C. Fernández-Córdoba, R. Ten-Valls, $\mathbb{Z}_{2}$-double cyclic codes, arXiv:1410.5604.

[5] J. Borges, C. Fernández-Córdoba, R. Ten-Valls, $\mathbb{Z}_{2} \mathbb{Z}_{4}$-additive cyclic codes, generator polynomials and dual codes, arXiv:1406.4425.

[6] H. Q. Dinh, S. R. LóPez-Permouth, Cyclic and negacyclic codes over finite chain rings, IEEE Trans. Info. Theory 50(8) (2004) 1728-1744.

[7] J. GaO, M. Shi, T. Wu And F. Fu, On double cyclic codes over $\mathbb{Z}_{4}$, Finite Fields and Their Applications 39 (2016) 233-250.

[8] A. R. Hammons, P. V. Kumar, A. R. Calderbank, N. J. A. Sloane, P. Solé, The $\mathbb{Z}_{4}$-Linearity of Kerdock, Preparata, Goethals, and Related Codes, IEEE Trans. Info. Theory 40(2) (1994) 301-319.

[9] G. Norton, A. Salagean, On the structure of linear and cyclic codes over finite chain rings Appl. Algebra Eng. Commun. Comput. 10 (2000) 489-506. 